\title{
Dynamical modelling and analysis of COVID-19 in India
}

\author{
R. Gopal ${ }^{1}$, V. K. Chandrasekar ${ }^{1, *}$ and M. Lakshmanan ${ }^{2}$ \\ ${ }^{1}$ Centre for Nonlinear Science and Engineering, School of Electrical and Electronics Engineering, SASTRA Deemed University, \\ Thanjavur 613 401, India \\ ${ }^{2}$ Department of Nonlinear Dynamics, School of Physics, Bharathidasan University, Tiruchirappalli 620014 , India
}

We consider the pandemic spreading of COVID-19 in India after the outbreak of the coronavirus in Wuhan city, China. We estimate the transmission rate of the initial infecting individuals of COVID-19 in India using officially reported data at the early stage of the epidemic with the help of the susceptible (S), exposed $(E)$, infected (I), and removed (R) population model, the so-called SEIR dynamical model. Numerical analysis and model verification are performed to calibrate the system parameters with official public information about the number of people infected, and then to evaluate several COVID-19 scenarios potentially applicable to India. Our findings provide an estimation of the number of infected individuals in the pandemic period of timeline, and also demonstrate the importance of governmental and individual efforts to control the effects and time of the pandemic-related critical situations. We also give special emphasis to individual reactions in the containment process.

Keywords: Containment process, COVID-19 pandemic, dynamical modelling, numerical analysis.

COVID-19, a disease caused by the severe acute respiratory syndrome coronavirus 2 (SARS-COV 2), was first identified in December 2019 in Wuhan, the capital of Hubei, China, and has since spread globally ${ }^{1}$. The World Health Organization (WHO) announced COVID-19 as an international public health emergency on 30 January 2020, and subsequently a pandemic on 11 March 2020 (ref. 2). The number of patients is growing exponentially and thousands of people are losing their lives in many a countries, almost every day due to COVID-19 (refs 3-5). The complexity of the situation can be realized from the fact that as on 15 May 2020 the number of coronavirus cases worldwide has reached a staggering $4,639,427$, with the number infected people being 2,564,442 and about 308,810 confirmed deaths have been reported due to this disease $^{6}$. Moreover, the outbreak has also spread to more than 200 countries $^{6}$. (Note added in revision: As on 20 November 2020, the number of coronavirus cases had reached as 46,403,652 with infected individuals being $12,079,415$ and 1,211,421 confirmed death cases.)

*For correspondence. (e-mail: chandru25nld@gmail.com)
Much work indicates that COVID-19 could spread from animals to humans (zoonotic) ${ }^{7}$. In addition, a rapid increase of COVID-19 infections shows that secondary transmission can occur through human-to-human contacts or through droplets transmitted by an infected person through coughing or sneezing ${ }^{4,7}$ or even when an infected person speaks to a non-infected one. With the above trend, this human-to-human spread of the disease is growing significantly almost everywhere in the world and the infection is rapidly increasing in many countries through local transmission ${ }^{4}$.

In India, infection due to COVID-19 was first identified on 2 March 2020. On 24 March 2020, the Government of India announced a 21-day country-wide lockdown as a preventive measure for the COVID-19 outbreak. The aim of the lockdown was to slow down the spread of the novel coronavirus, allow the Government to follow a multi-pronged strategy to add more beds in its hospitals network, increase the production of the COVID-19 test kits and personal protective equipment (PPEs) for health workers, etc. The government frequently used various platforms to keep the public aware of COVID-19. In India, conditions like very high population density in urban areas, unavailability of vaccines and inadequate data about disease transmission also make it a difficult task to adequately fight the disease.

Mathematical simulations have often been used to forecast the effects of various epidemics and also to test the efficacy of different prevention approaches in reducing disease burden ${ }^{8}$. Recently, with regard to the COVID19 pandemic, considerable research has been carried out using actual occurrence from the impacted countries, analysing various aspects of the epidemic, as well as assessing the impact of preventive approaches adopted in order to limit the epidemic in the countries concerned ${ }^{9}$. In particular, various kinds of dynamical models have been employed, essentially considering nonlinear governing equations. For instance, the nature of human coronavirus infection and defining contact between human cells and the virus have been described in Rihan et al. ${ }^{10}$. A statistical model for estimating virus transmission, taking into account a condensed version of the bats-hosts-reservoirpeople transmission model, known as the reservoirpeople model was reported by Chen et al. ${ }^{11}$. 
The susceptible-exposed-infectious-recovered (SEIR) model is an important tool for tackling coronavirus transmission statistical simulations. Lin et al. ${ }^{3}$ have proposed a method to develop analytical models for the COVID-19 outbreak in Wuhan, taking into account human behavioural responses and government decisions, such as holiday extension, travel restriction, hospitalization and quarantine ${ }^{3}$. Some recent studies use numerical simulations and provide a reliable real-time forecast of COVID-19 cases in various countries with the help of this mathematical model. For instance, Savi et al. ${ }^{12}$ have obtained the general transmission of the novel coronavirus to test various scenarios of propagation in different countries, taking into account model testing of the evolution of infected populations in China, Italy, Iran and Brazil based on government and individual reactions.

In this study, we consider the situation in India starting from the initial outbreak period and fit the SEIR model to daily infected cases reported between 2 March and 15 May 2020. We estimate the basic transmission rate of COVID-19 in the initial stage of the epidemic between 2 and 24 March 2020. Further, the general propagation of the novel coronavirus is also studied to evaluate different scenarios of the various propagation. In addition, the model verification takes into consideration the evolution of the infected population and simulates different scenarios based on the rate of transmission and the governmental and, especially, individual reactions. Finally, it proposes potential evolution of the spread and possible mitigations, specifically emphasizing the significance of individual reactions at the societal level during the pandemic period, which includes both lockdown and various unlock periods.

Appendix 1 provides details of this study on the number of infected individuals during the lockdown and unlock periods starting from 25 March to 31 October 2020 and further up to 27 December 2020.

\section{Dynamical model}

Lin et $a l .^{3}$ have proposed SEIR model to explain the coronavirus disease ${ }^{3}$. This was inspired by the original influenza model of He et al. ${ }^{13,14}$. The SEIR model also has two supplementary terms: $D$ is a public perception of risk with respect to serious cases and deaths and $C$ is the number of recorded and unreported incidents. In addition, $S$ is the susceptible population, $E$ the population exposed, $I$ the currently infectious population (excluding the recovered and death cases) and $R$ is the population removed which includes both cases recovered and deaths. The simplified version of the governing equations takes account of the interaction between all these populations, and is represented by the following set of coupled nonlinear differential equations ${ }^{3}$ :

$$
\dot{S}=-\beta(t) \frac{S I}{N},
$$

$$
\begin{aligned}
& \dot{E}=\beta(t) \frac{S I}{N}-\sigma E, \\
& \dot{I}=\sigma E-\gamma I, \\
& \dot{R}=\gamma_{R} I, \\
& \dot{D}=d_{\gamma} I-\lambda D, \\
& \dot{C}=\sigma E,
\end{aligned}
$$

where $\gamma$ is the mean infectious period, $\gamma_{R}$ the delayed removed period, which denotes the relation between the removed and infected populations, $\sigma$ the mean latent period, $d$ denotes the proportion of severe cases and $\lambda$ is the mean duration of public reaction ${ }^{3,4,12}$.

In eqs (1)- $(6), \beta(t)$ denotes the transmission rate function which incorporates the impact of governmental action $(1-\alpha)$ and individual actions which is denoted by the function $(1-D / N)^{k}$ (refs 3,12$)$. Here, the parameter $k$ defines the intensity of individual reactions, which is measured on a scale of 0 to $10^{5}$ with a normal value of 1117.3 obtained from previous and recent epidemic and pandemic studies ${ }^{3,13}$. We also assume that the effect of governmental action is different during various lockdown periods. Therefore, the transmission rate $\beta(t)$ is defined as

$$
\beta(t)=\beta_{0}(1-\alpha)\left(1-\frac{D}{N}\right)^{k}
$$

The value of $\beta_{0}$ is derived by assuming that the basic reproduction number is $R_{0}=\beta_{0} / \gamma$, which measures the average number of new infections generated by each infected person. The values of the system parameters are mentioned in Table 1, based on the information deduced from the literatures ${ }^{3,4,12,13,15}$.

The above parameters must be modified for each state/country, which is important for COVID-19 analysis. In general, the physical meanings of the parameters are based on a variety of facts, identifying which is a difficult $\operatorname{task}^{3}$. It should be also pointed out in this regard that the real data have spatial aspects that are not covered by the above set of governing equations. Consequently, this kind of study is a sort of average activity that needs careful adjustment to suit real data as followed by earlier studies $^{3,12}$.

In the present study, we use step-like functions to define certain parameters that allow for a proper representation of different scenarios, especially the rate of transmission. It is also important to remember that all governmental or individual decisions have a delayed impact on the dynamics of the system. Further, virus mutation is an important factor related to the definition of 
Table 1. Summary table of the parameters discussed in eqs (1)-(7)

\begin{tabular}{lll}
\hline Parameter & \multicolumn{1}{c}{ Description } & \multicolumn{1}{c}{ Value/remarks/reference } \\
\hline$N_{0}$ & Initial number of population & India populations ${ }^{16}$ \\
$S_{0}$ & Initial number of susceptible population & $0.9 N_{0}$ (constant) \\
$E_{0}$ & Exposed persons for each infected person & $20 I_{0}$ (assumed) \\
$I_{0}$ & Initial state of infected persons & 3 (ref. 15) \\
$\alpha$ & Governmental action strength & Varied during each lockdown period \\
$k$ & Intensity of individual reactions & 1117.3 (refs 3, 12) \\
$\sigma^{-1}$ & Mean latent period & 3 (days) \\
$\gamma^{-1}$ & Mean infectious period & 5 (days) \\
$d$ & Proportion of severe cases & 0.2 \\
$\lambda^{-1}$ & Mean duration of public reaction & 11.2 (days) \\
\hline
\end{tabular}

COVID-19 dynamics which can affect the reaction of the system significantly; however, this is not discussed in the present study ${ }^{12}$. Next, let us treat the COVID-19 dynamics considering two different objectives. To start with, we identify the initial value of transmission rate with data from initially infected people, and then examine various scenarios for the COVID-19 situation in India using different transmission rates and governmental and individual action strengths.

\section{Data analysis with numerical model}

As a first phase of the established study, model verification was performed using information available from India's COVID-19 tracker and worldometers.info ${ }^{6}$. We followed the SEIR implementation methodology described for various countries by Savi et al. ${ }^{12}$. The model employed for simulations has the total population of India at $N \approx 139 \times 10^{7}$ (ref. 16), and three infected COVID-19 cases confirmed as on 2 March 2020. The initial state is taken to be with $I_{0}=3$ and a susceptible initial population is assumed to be $S_{0}=0.9 \mathrm{~N}$. Another information needed for the model is the number of individuals exposed to each infected person. Each infected individual is believed to have the potential to infect a further 20 individuals, $E_{0}=20 I_{0}$ (refs 3,12). Initially, we started with the estimation of the initial transmission rate.

\section{Estimation of initial transmission rate}

To start with, we noted that intensified precautionary measures to curb the spread of COVID-19 has been carried out in India. In particular, when patients were found at a location, the Committee of Physicians and Experts checked those people who had contact with these patients. Then, both the patients and the contaminated people were quarantined in a hospital or other isolated areas. In the data published in India, in the initial few days, the active cases were automatically moved to quarantined cases ${ }^{17}$. Therefore, the active cases correlate after a few days with the omitted quarantined cases. From the recorded data we can match real infected individuals (excluding quarantined people) as a function of time at the early stage of disease spreading barring the initial quarantined people ${ }^{18}$.

In order to solve the model (1), we considered the susceptible population to be close to the overall population in the early stages of transmission of the disease $\mathrm{e}^{20}$. Then we can rewrite the dynamic equation of the epidemic with $S / N \approx 1$.

In this case, from eqs (2) and (3), the equation for the presently infectious population (which excludes the recovered and death cases) becomes the second-order linear ordinary differential equation of damped linear type

$$
\ddot{I}+(\gamma+\sigma) \dot{I}-\sigma(\beta-\gamma) I=0
$$

By integrating the above equation, we obtain the solution as $^{19}$

$$
I(t)=I_{01} e^{-\frac{1}{2}\left(\gamma+\sigma-\sqrt{\left.(\gamma-\sigma)^{2}+4 \sigma \beta\right) t}\right.}+I_{02} e^{-\frac{1}{2}\left(\gamma+\sigma+\sqrt{\left.(\gamma-\sigma)^{2}+4 \sigma \beta\right) t}\right.},
$$

where $I(0)=I_{01}+I_{02}$ is the initial number of infected people. Now the curve is fitted with the initial real infectious population data available in ref. 6 . Further we identify an exact fit with the curve $3 e^{0.473 t}$. From this we obtain the parameter values as $I_{01}=3, I_{02}=0$ and the initial value of $\beta=0.56$. This $\beta$ value can be considered as the initial transmission rate $\beta_{0}$ in eq. (7). It gives the reproduction number $R_{0}=\beta_{0} / \gamma=2.8$. One may note that a few recent studies suggest a reproduction rate of $R_{0}$ about 2.52 for the first 22 days before lockdown, and then identify the value of $R_{0}$ to vary between 1.9 and 3.0 in various places in India, due to variation of transmission rates $^{15,18,20}$. However, in our analysis, the estimation of $R_{0}$ from the model is confirmed with real data of daily infected individuals (excluding recovered and death cases). Figure 1 represents the curve fit of infected people as a function of time at the early stage of disease spread in India. 


\section{Verification through simulations}

The first scenario for model verification based on the results of India is presented here. It should be noted that our analysis considers all infected cases in the entire country, and it is not restricted to any specific state or place. Table 1 provides the parameters which are used in the model. These should be treated as average values since they are appropriate for the country as a whole. Initially, we started with the value of transmission rate $\beta_{0}=0.56$. The value attributable to governmental action strength was also considered as different during the periods before and after lockdown. Figure 2 shows the transmission rate $\beta(t)$, before and after lockdown period with respect to various governmental action strengths. One may note that an important constraint on the parameter $\beta(t)$ is that this variable should be a step-like function with time due to the impact of governmental action strength. Therefore, using the daily COVID-19 incidence data, numerical simulation was carried out for the model (7) with the value of $\beta_{0}=0.56$. Figure 3 shows the evolution of the number of infected individuals (excluding both

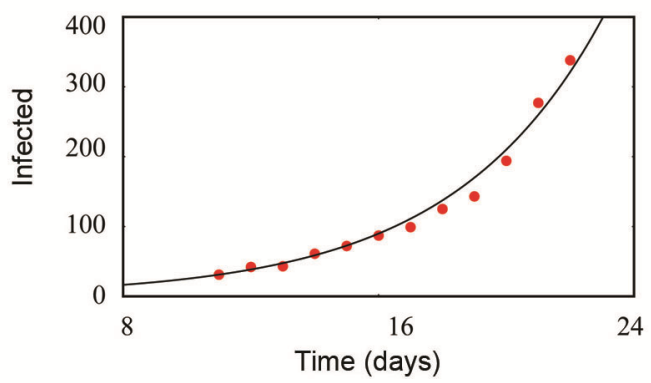

Figure 1. The number of infected individuals (excluding the number of initial quarantined people) after the first few days of outbreak on 2 March 2020. Data were fitted by the nonlinear least-square fit for India. The solid black line is for fitting data and red circles denote real data of the number of infected individuals.

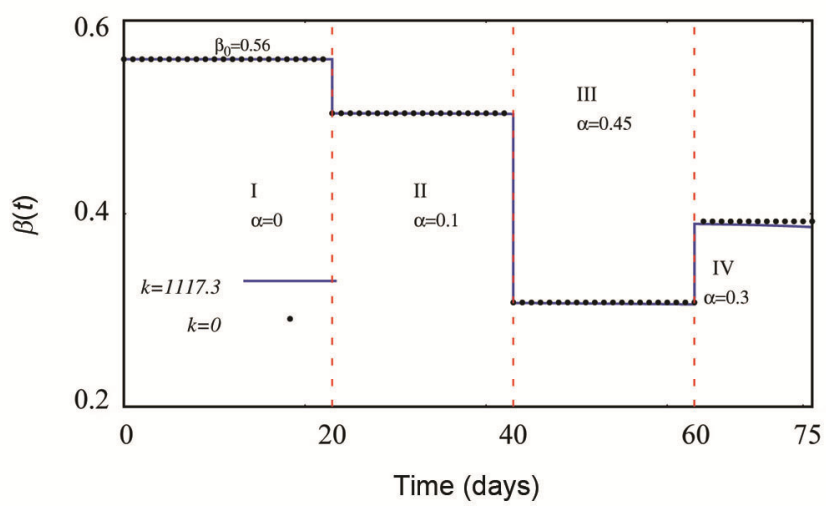

Figure 2. Variation of transmission rate considered in our model eqs (1)-(6) as a function of time with the impact of $\alpha$ in eq. (7) with respect to initial transmission value 0.56 . The continuous line is for intensity of individual reactions $k=1117.3$ and the dotted line is for $k=0$. recovered and death cases), indicating a strong agreement between simulation and actual data. The infected people regions are marked as I, II, III and IV and they correspond to the periods of 2-24 March (before lockdown), 25 March-14 April (first lockdown), 15 April-3 May (second lockdown) and 4-17 May (third lockdown) respectively.

It is apparent that our estimation of the number of infected individuals in model eqs (1)-(7), with basic transmission rate, system parameters and different governmental action strengths up to the end of the second lockdown period starting from the initial outbreak (2 March-3 May) shows good agreement with the actual data of infected individuals (Figure $3 a$ ). This study helps predict newly infected individuals in the near future. For instance, we have chosen low values of governmental action strengths, $\alpha=0.3$ and 0.4 , in the third lockdown period (due to minimum relaxation given by the government, when certain activities were permitted/restricted in each zone) compared to $\alpha=0.45$ during the second lockdown period. Our study predicts the number of infected individuals reasonably well with the real data for $\alpha=0.4$ in the region IV (black curve in Figure $3 b$ ). Note that in Figure 3 , we have also included the strength of individual reactions at a rather low value of $k=1117.3$ (see eq. (7)), but
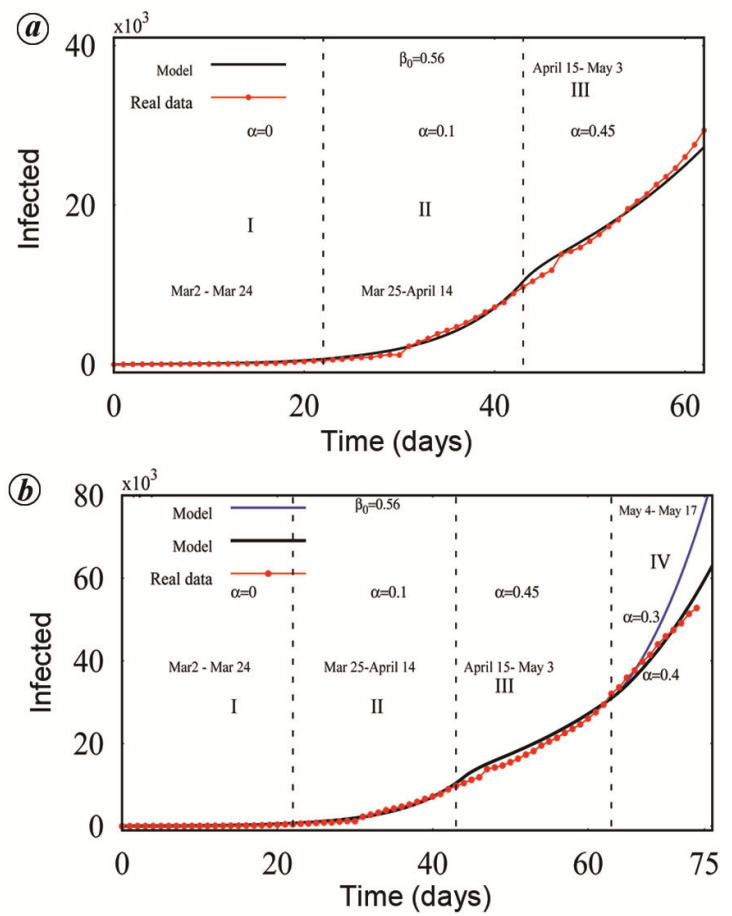

Figure 3. Numerical simulation of the number of infected individuals (excluding both recovered and dead) with initial transmission rate taken as $\beta_{0}=0.56$ and for different governmental action strengths before and after the lockdown period. The prediction of the mathematical model (continuous blue and black curves) compared with actual data (red dotted curve) for daily infections in India up to (a) 3 May 2020 and (b) 17 May 2020 is depicted. The strength of individual reactions is considered as $k=1117.3$ and the remaining parameters are taken from Table 1. 
Table 2. Sample summary of actual data of COVID-19 pandemic in India and predicted infected people from the SEIR model given by eqs (1)-(7)

\begin{tabular}{lcc}
\hline & $\begin{array}{c}\text { Number of infected people } \\
\text { Date }\end{array}$ & \\
\hline $04 / 03 / 2020$ & SEIR model $(I(t))$ \\
$10 / 03 / 2020$ & 58 & 29 \\
$24 / 03 / 2020$ & 486 & 84 \\
$2 / 04 / 2020$ & 2280 & 679 \\
$11 / 04 / 2020$ & 7189 & 2249 \\
$14 / 04 / 2020$ & 9735 & 7103 \\
$15 / 04 / 2020$ & 10,440 & 10,417 \\
$19 / 04 / 2020$ & 14,202 & 11,564 \\
$23 / 04 / 2020$ & 17,306 & 14,660 \\
$24 / 04 / 2020$ & 18,171 & 17,575 \\
$25 / 04 / 2020$ & 19,519 & 18,370 \\
$03 / 05 / 2020$ & 29,339 & 19,198 \\
$10 / 05 / 2020$ & 43,980 & 27,261 \\
$13 / 05 / 2020$ & 49,104 & 43,910 \\
$14 / 05 / 2020$ & 51,379 & $55,958(\alpha=0.3) ; 46,368(\alpha=0.4)$ \\
$15 / 05 / 2020$ & 52,773 & $60,668(\alpha=0.3) ; 49,073(\alpha=0.4)$ \\
\hline
\end{tabular}
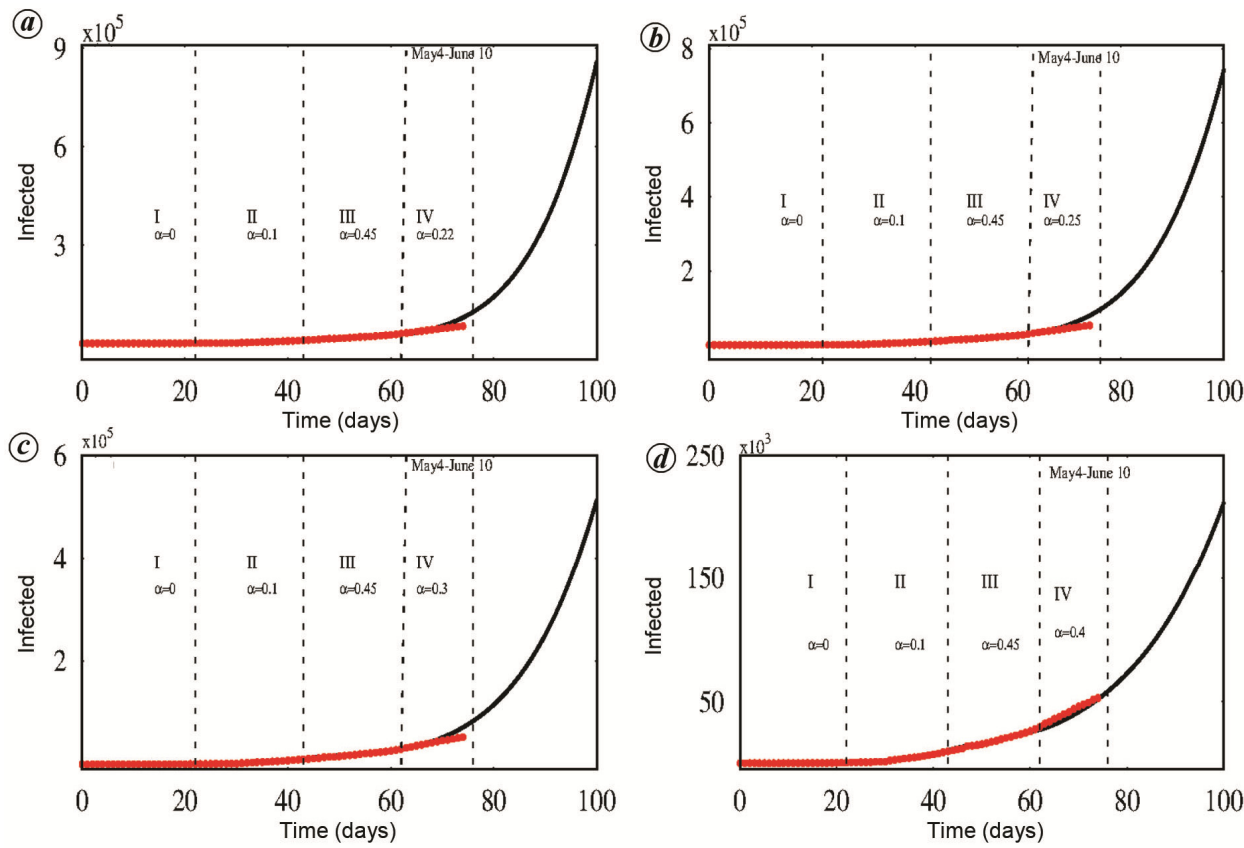

Figure 4. Numerical simulation of the evolution of the number of infected individuals with the initial transmission rate $\beta_{0}=0.56$. The red curve denotes actual data for daily infections (excluding recovered and death cases) in India up to 15 May 2020 . Note that this simulation (IV) shows the rate of newly infected population after 3 May with respect to the values of governmental action strength in eq. (7) with (a) $\alpha=0.22,(\boldsymbol{b}) \alpha=0.25,(\boldsymbol{c}) \alpha=0.3$ and $(\boldsymbol{d}) \alpha=0.4$. The strength of individual reactions considered is a rather low value of $k=1117.3$ and the remaining parameters are taken from Table 1 .

the contribution due to this term is only minimal. Table 2 provides a sample summary of the actual infected people in India ${ }^{6}$, and prediction of infected individuals from model eqs (1)-(6).

\section{Role of governmental action and individual reactions}

We also analyse the possibility of impact of prevention approaches in reducing new cases infected with COVID19 using the above mathematical model, after 3 May
2020. For instance 4 May 2020-10 June 2020, and beyond (region IV, Figure $4 a-d$ ). Strategies for prevention include mechanisms such as lockdown, information campaign by newspapers and television, adequate hand sanitation, social distancing, etc. which result in slowing down the COVID-19 transmission process. These strategies of prevention as modelled in terms of the parameters $\alpha$ and $k$ in eq. (7), imply that there will be a reduction in the transmission rate $\beta(t)$. Now, we start with the efficiency of prevention by varying governmental action strength alone, keeping $k$ at a fixed low strength 
$(k=1117.3)^{3}$. For $\alpha=0.22$ and $\alpha=0.25$, it is observed that the number of infected individuals will peak around $8 \times 10^{5}$ and $7 \times 10^{5}$ respectively by 10 June (Figure $4 a$ and $b$ ). If the strength is increased $(\alpha=0.30)$, the peak of infected/active people cases may decrease and occurrence of the peak is shifted down to $5 \times 10^{5}$ on 10 June (Figure $4 c$ ). Now, we consider a further increased value of governmental action strength $(\alpha=0.4)$, and the newly infected cases can decrease and reach around $2.5 \times 10^{5}$ by 10 June (Figure $4 d$ ).

Analysing the results, the present dynamical model clearly shows that when the value of $\alpha$ is reduced, new infected cases continue to quickly increase, while a larger value of $\alpha$ decreases the infected cases to a considerable extent. However, the latter will not help in reducing the infection to approach zero, unless perhaps $\alpha$ approaches a value close to unity. Moreover, it is not practical to keep increasing the governmental action to a higher and higher level even in the fourth lockdown period and further due to the necessity of opening up the economic front for survival of the nation. On the other hand, with relaxation of governmental action over time, particularly in the fourth lockdown period and subsequent periods, the above prevention method alone will help in the eradication of the disease.

In Figure 4, we have shown simulations based on various governmental action strengths with fixed low value
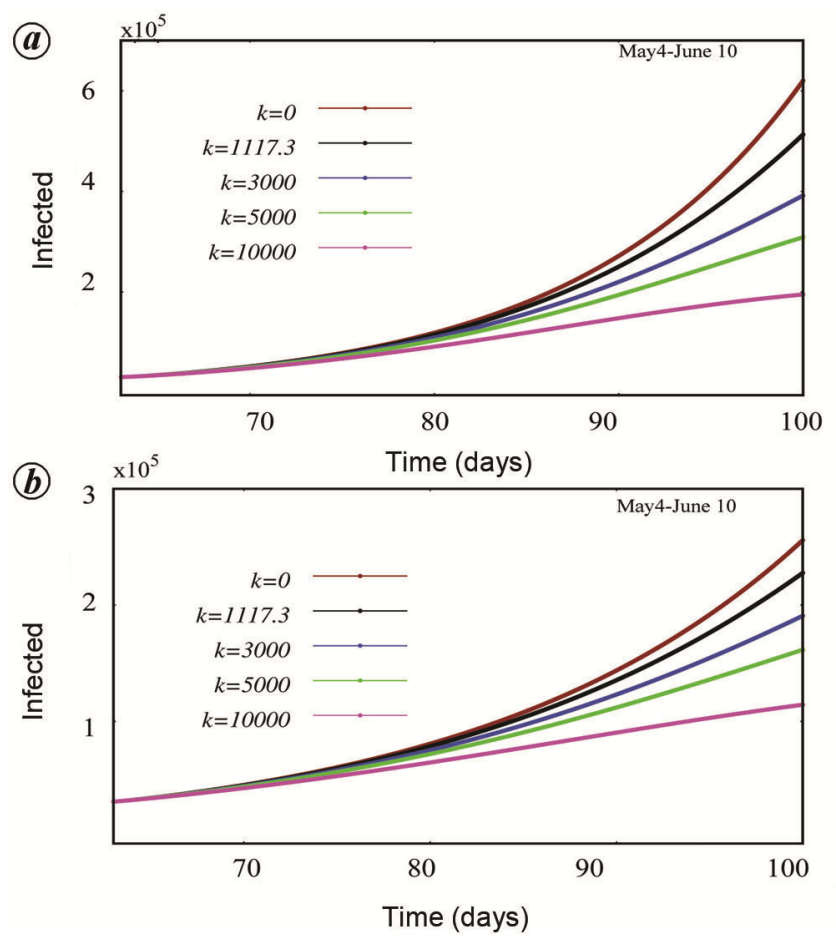

Figure 5. Numerical simulation of the evolution of the number of infected individuals with the specific value of initial transmission rate $\beta_{0}=0.56$, after 3 May (expanded view of IV in Figure $4 c$ and $d$ ) with respect to different values of intensity of individual responses $k$ in eq (7) for different values of governmental action strength with (a) $\alpha=0.3$ and $(\boldsymbol{b}) \alpha=0.4$. The remaining parameters are taken from Table 1 . of intensity of individual reactions $(k=1117.3)$ in eq. (7). Individual reactions or behaviour can include social distancing, personal hygiene, healthy habits, avoiding crowded places, etc. ${ }^{2}$. It will also include alerting fellow citizens to wear masks, follow personal hygiene and social distancing, political and social organizations urging fellow citizens to follow social norms, and so on. Now, we also address the importance of individual reactions with low value of governmental action strength $\alpha=0.3$ and $\alpha=0.4$ (region V of Figure 5, which corresponds to the period beyond 18 May 2020). We observe from Figure 5 that the number of infected people increases for no action of individual response (for $k=0$ ), while the number of infected people decreases when the value of individual response increases to $k=3000,5000$ and 10,000 . We also learn that individual behavioural responses are important along with governmental action.

In the above scenario, we further considered different low values of governmental action strength $\alpha$ in region IV of Figure $4 a-d$ (3 May-10 June 2020) and various values of intensity of individual reactions in the same region in Figure $5 a$ and $b$. The corresponding figures show that the disease continues to infect more and more people due to low value of governmental action strength, but it can be controlled with respect to appropriate individual reactions. In order to break the chain of infection spread and to get more control on infected individuals,
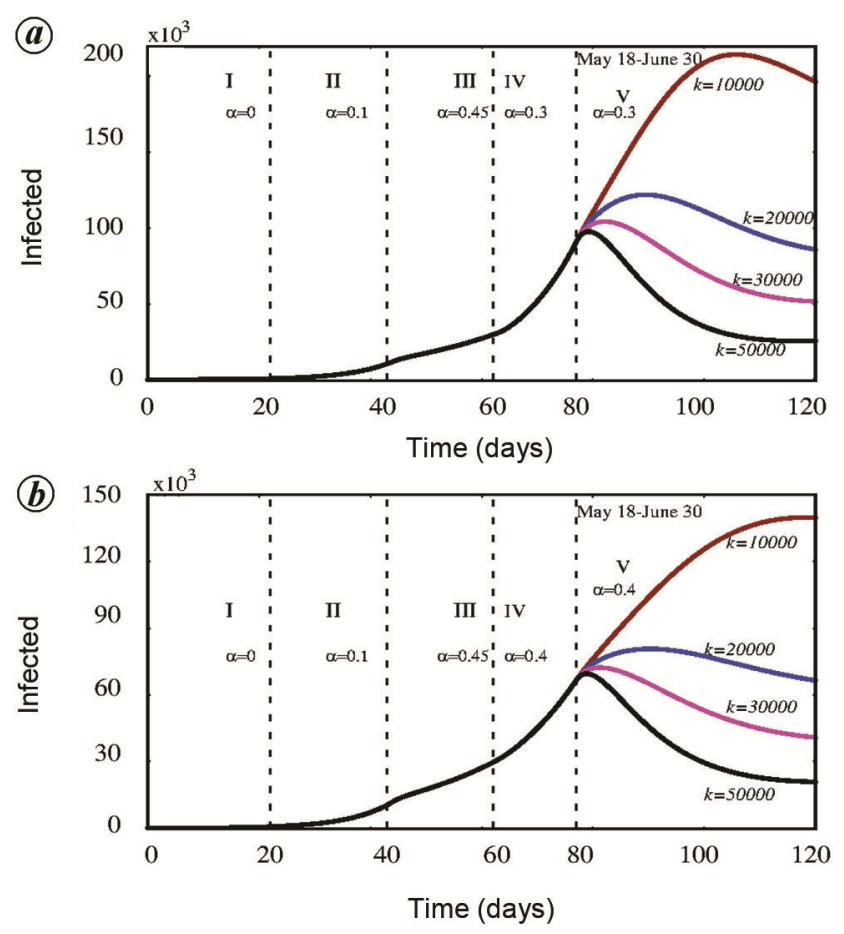

Figure 6. Numerical simulation of the evolution of the number of infected individuals for the initial transmission rate $\beta_{0}=0.56$ and $k=1117.3$ (I-IV). Note that this simulation shows the rate of infected people after 17 May (V) with respect to different values of individual reactions in eq. (7) with (a) $\alpha=0.3$ and (b) $\alpha=0.4$. The remaining parameters are taken from Table 1. 
one may choose immediate action of the individual reaction response after the third lockdown period, that is, range IV, and consider appropriate values of $\alpha$ and $k$ in the time window $\mathrm{V}$ in Figure 6 . For $\alpha=0.3$ or 0.4 and $k=10,000$ or 20,000 the newly infected cases tend to decrease within a few weeks, after 18 May (Figure $6 a$ and $b$ ). If the individual reaction is increased further $(k=30,000$ and 50,000), we see that the disease can be effectively eradicated within 1-2 months from 18 May 2020 (Figure $6 a$ and $b$ ).

Based on our analysis in Figures 4-6, we find that if we introduce appropriate values of individual reaction strength $k$, then even for low values of governmental action, the reduction can become substantially impressive and one can approach a regime of complete control of the disease in a reasonably short period in the absence of appropriate vaccination.

\section{Conclusion}

On 3 May 2020, the total number of active infected cases registered for COVID-19 and deceased cases in India was 29,339 and 1391, on 15 May it was 52,773 and 2753 and on 4 November 2020 it was at 533,787 and 123,611 respectively ${ }^{17}$. This rise in the active infected cases has occurred after some minimal relaxation in the government lockdown, and several hundred new cases are being reported each day from different locations across India. Our study analysed the effect of lockdown days on the spread of COVID-19 disease in the country. Therefore, predictive mathematical/dynamical models can also provide useful insights to strengthen our understanding of COVID-19 transmission and control.

In this study, we considered the dynamical SEIR model, and estimated the initial rate of COVID-19 transmission by considering the initially infected people in India. In addition, a verification procedure was performed with respect to different transmission rates based on the data available from India. Our findings also indicate that the government and in particular individual efforts are important in reducing infected populations and also the overall time-period of the epidemic. In addition, such epidemic mathematical models and their predictive simulations are valuable resources which can be helpful for public health planning and in governmental as well as individual acts. Further, this study also shows that the COVID-19 pandemic can be suppressed by a lockdown.

Our model and current data seem to indicate that the confirmed infected individuals continue to grow in India every day, in spite of rapid response by the government to the pandemic through various quarantine measures, nationwide lockdown and risk-based zoning when the individual reaction rate $(k)$ is chosen to be low. However, we find that for appropriate increased values of individual reactions, even with low governmental action strength, there can be dramatic reduction in the total number of infected people. Depending upon the increased individual contribution, the disease can be effectively controlled in a rather short period. It is then imperative that the society as a whole contributes its might by simple social measures, besides appropriate governmental action. These combined efforts can help in total control of the disease in a short period in India and perhaps elsewhere as well.

Appendix 1. Study of infected individuals during the lockdown and unlock periods.

In order to get a clear insight on the estimation of our prediction, Figure A1 shows the rate of infected individuals with respect to the timeline of various lockdown and unlock periods. It shows that various intervention strategies (lockdown, spreading awareness programme, public reaction, proper hand sanitization, etc.), including measures like governmental action strength $\alpha$ and intensity of individual reactions $k$ play an important role in controlling the number of infected individuals in India.

Starting from the initial date of outbreak, we consider a period of 300 days as the timeline and study the impact of various interventions during this period. In terms of the actual dates, we consider the time period between 2 March and 27 December 2020.

Our study shows that with comparatively low values of the parameters $\alpha$ and $k$, the time of occurrence of the peak of the outbreak occurred during the unlock period 4.0 (region IX in Figure A1). If the strength of the intensity of individual reactions increases further from $k=2500$ in region IX to 3500 in region $\mathrm{X}$ and then to 6000 and 10,000 or even 20,000 in the region XI in the figure, the number of infected individuals decreases rather quickly and can reach a minimum at the end of

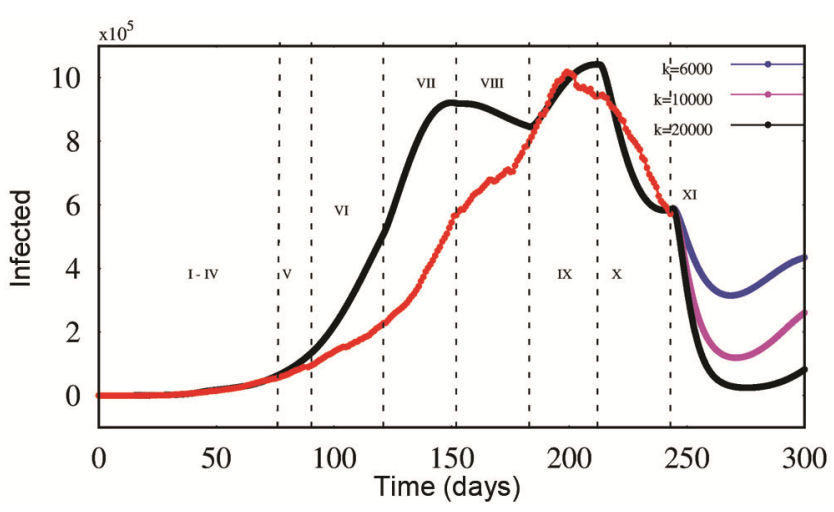

Figure A1. Numerical simulation of infected people with initial transmission $\beta_{0}=0.56$. The red curve denotes actual data from refs 6 , 19 for daily infections in India up to 31 October 2020. Note that the simulation (I-XI) shows the rate of infected people in the timeline from 2 March 2020 to 27 December 2020 with respect to various values of $\alpha$ and individual reactions $k$ as given in Table A1. If the strength of the individual reactions $k$ is increased further (after 31 October 2020), the number of infected individuals may decrease and come to an end in a short period of time (black curve in XI). 
Table A1. Values of government action strength $\alpha$ and intensity of individual reactions $k$ used during no lockdown (No LD), lockdown (LD) and unlock (UL) periods in eq. (7)

\begin{tabular}{llcccc}
\hline Phase & Timeline (2020) & Figure & Region & $\alpha$ Value & $k$ Value \\
\hline No LD & 2 March-24 March & $3 b$ & I & 0.0 & 1117.3 \\
LD-1 & 25 March-14 April & $3 b$ & II & 0.1 & 1117.3 \\
LD-2 & 15 April-3 May & $3 b$ & III & 0.45 & 1117.3 \\
LD-3 & 4-17 May & $3 b$ & IV & 0.40 & 1117.3 \\
LD-4 & 18-31 May & A1 & V & 0.40 & 2000 \\
UL-1 & 1-30 June & A1 & VI & 0.38 & 2100 \\
UL-2 & 1-31 July & A1 & VII & 0.30 & 2200 \\
UL-3 & 1-31 August & A1 & VIII & 0.25 & 2300 \\
UL-4 & 1-30 September & A1 & IX & 0.11 & 2500 \\
UL-5 & 1-31 October & A1 & X & 0.10 & 3500 \\
UL-6 & 1 November- & A1 & XI & 0.10 & 6000 (blue) \\
& & A1 & XI & 0.10 & 10,000 (pink) \\
& & A1 & XI & 0.10 & 20,000 (black) \\
\hline
\end{tabular}

December 2020. (The increased value of individual reactions $k$ may be attributed to increased awareness among people and more number of individuals following basic hygiene, etc.) Our results seem to agree well with the recent COVID-19 data on India ${ }^{2,17}$. Our study also shows that when the value of $k$ is decreased further in the forthcoming period in the XI window region in Figure A1, the new cases tend to increase. This shows the possibility of rapid increased occurrence of infected individuals (blue and pink curves in region XI, Figure A1).

1. Cohen, J. and Normile, D., New SARS like virus in China triggers alarm, Science, 2020, 367, 6475.

2. WHO, 2020; https://www.who.int/emergencies/diseases/novelcoronavirus-2019/technical-guid (accessed on 16 May and 5 November 2020).

3. Lin, Q. et al., A conceptual model for the coronavirus disease 2019 (COVID-19) outbreak in Wuhan, China with individual reaction and governmental action. Int. J. Infect. Dis., 2020, 93, 211 216.

4. Li, Q. et al., Early transmission dynamics in Wuhan, China, of novel coronavirus-infected pneumonia. New Engl. J. Med., 2020, 382, 1199; doi:10.1056/NEJMoa2001316.

5. Ferguson, N. M. et al., Impact of non-pharmaceutical interventions (npis) to reduce COVID-19 mortality and healthcare demand. Imperial College COVID-19 Response Team, London, UK, 2020; doi:10.25561/77482.

6. https://www.worldometers.info/coronavirus/country; https://www. covid19india.org (accessed on 16 May and 5 November 2020).

7. Rothan, H. and Byrareddy, S., The epidemiology and pathogenesis of coronavirus disease (COVID-19) outbreak. J. Autoimmun., 2020, 109, 102433; doi:10.1016/j.jaut.2020.102433.

8. Kermack, W. O. and McKendrick, A. G., Contributions to the mathematical theory of epidemics. Bull. Math. Biol., 1991, 53, 33.

9. arXiv.org, https://arxiv.org; bioRxiv.org, https://www.biorxiv.org.

10. Rihan, F. A., Al-Salti, N. S. and Anwar, M. N. Y., Dynamics of coronavirus infection in human, AIP Conf. Proc., 2018, 1982, 020009; doi: $10.1063 / 1.5045415$.

11. Chen, T. M., Rui, J., Wang, W. P., Zhao, Z. Y., Cui, J. A. and Yin, L., A mathematical model for simulating the phase-based trans- missibility of a novel coronavirus. Infect. Dis. Poverty, 2020, 9, 24; doi:10.1186/s40249-020-00640-3.

12. Savi, P. V., Savi, M. A. and Borges, B., A mathematical description of the dynamics of coronavirus disease (2019): (COVID-19): a case study of Brazil, 2020; arXiv:2004.03495v2.

13. He, D., Dushoff, J., Day, T., Ma, J. and Earn, D. J. D., Inferring the causes of the three waves of the 1918 influenza pandemic in England and Wales. Proc. R. Soc. London Ser. B, 2013, 280, 20131345; doi:10.1098/rspb.2013.1345.

14. He, D., Ionides E. L. and King, A. A., Plug-and-play inference for disease dynamics: measles in large and small populations as a case study. J. R. Soc. Interface, 2010, 7, 271-283; doi:10.1098/rsif. 2009.0151.

15. Das, S., Prediction of COVID-19 disease progression in India. 2020; arXiv:2004.031471v1.

16. https://www.statisticstimes.com/demographics/population-of-india. php (accessed on 16 May 2020).

17. https://www.mygov.in/covid-19 (accessed on 16 May and 5 November 2020).

18. Chae, S. Y. et al., Estimation of infection rate and prediction of initial infected individuals of COVID-19. 2020; arxiv.org/ pdf $/ 2004.12665$.

19. Lakshmanan, M. and Rajasekar, S., Nonlinear dynamics: Integrability Chaos and Patterns, Springer-Verlag, Berlin, Germany, 2003.

20. Liu, Y., Gayle, A. A., Smith, A. W. and Rocklow, J., The reproductive number of COVID-19 is higher compared to SARS coronovirus. J. Travel Med., 2020, 27(2), 1-4; doi:10.1093/ $\mathrm{jtm} / \mathrm{taaa} 021$.

21. MoHFW, Corona virus disease 2019 (COVID-19). Ministry of Health and Family Welfare, Government of India, 2020; https://www.mohfw.gov.in

ACKNOWLEDGEMENTS. V.K.C. acknowledges a research project sponsored by SERB-DST-MATRICS Grant No. MTR/2018/000676. M.L. thanks the Department of Science and Technology, New Delhi for the award of SERB Distinguished Fellowship under Grant No. SB/DF/ $04 / 2017$.

Received 19 May 2020; revised accepted 7 December 2020

doi: $10.18520 / \mathrm{cs} / \mathrm{v} 120 / \mathrm{i} 8 / 1342-1349$ 